УДК 621.313 .323

\title{
ПРИМЕНЕНИЕ МЕТОДА НЕЛДЕРА-МИДА ДЛЯ ОПТИМИЗАЦИИ ОДНОИМЕННОПОЛЮСНОГО СИНХРОННОГО ДВИГАТЕЛЯ ДЛЯ КАРЬЕРНОГО САМОСВАЛА
}

\author{
Прахт Владимир Алексеевич1', \\ va.prakht@urfu.ru
}

\section{Дмитриевский Владимир Александрович1, vladimir.dmitrievsky@urfu.ru}

\author{
Анучин Алексей Сергеевич², \\ anuchinas@mpei.ru \\ Казакбаев Вадим Маратович1, \\ vadim.kazakbaev@urfu.ru \\ 1 Уральский Федеральный Университет, \\ Россия, 620002, г. Екатеринбург, ул. Мира, 19. \\ 2 Национальный исследовательский университет «МЭИ», \\ Россия, 111250, г. Москва, ул. Красноказарменная, 14.
}

\begin{abstract}
Актуальность исследования обусловлена возрастающей потребностью применения карьерных самосвалов с дизельэлектрическим (гибридным) приводом для разработки полезных ископаемых. Улучшение рабочих и стоимостных характеристик электропривода карьерных самосвалов способствует уменьшению затрат при разработке полезных ископаемых.

Цель: поиск эфффективного подхода для оптимизации одноименнополюсного синхронного двигателя для привода колес карьерного самосвала, позволяющего решить проблему высокой потребности в вычислительных ресурсах при расчете трехмерной модели магнитного поля двигателя; выработка рекомендаций к проектированию одноименнополюсного синхронного двигателя с обмоткой возбуждения на статоре; оптимизация с целью уменьшения потерь мощности и максимального тока двигателя при заданной тяговой характеристике привода для снижения номинального тока и стоимости инверторного полупроводникового модуля электропривода карьерного самосвала с рассматриваемым типом двигателя.

объекты: конструкция шестиполюсного девятисразного одноименнополюсного синхронного двигателя с обмоткой возбуждения на статоре мощностью 370 кBm.

Методы: безградиентный метод оптимизации, математическое моделирование, двухмерный метод конечных элементов, метод схем замещения.

Результаты. Предложен новый, эффрективный с точки зрения точности расчета характеристик и вычислительных затрат, подход к оптимизации одноименнополюсного синхронного двигателя. В результате проведенной оптимизации потери в двигателе и максимальный ток, потребляемый двигателем от инвертора, были значительно снижены. Достигнутое снижение максимального тока позволяет снизить стоимость полупроводниковых модулей инвертора в 1,4 раза, а также позволяет снизить на ту же величину переменную составляющую тока звена постоянного тока инвертора.
\end{abstract}

\section{Ключевые слова:}

Карьерный самосвал, метод Нелдера-Мида, одноименнополюсный синхронный двигатель, оптимальное проектирование, тяговый двигатель, тяговый привод .

\section{Введение}

Значительная часть работы по перевозке породы при разработке полезных ископаемых осуществляется с помощью карьерных самосвалов грузоподъемностью 90-450 т. Они имеют преимущества как перед конвейерным транспортом в условиях перемещения горных пород с разными физико-механическими свойствами, так и перед железнодорожным транспортом, позволяя упростить процесс отвалообразования. Одной из особенностей карьерных самосвалов является применение силовых установок больших мощностей, до 4600 л. с. Это влечет за собой неизбежное усложнение трансмиссии. Привод на колеса осуществляется от двигателя внутреннего сгорания через гидромеханическую коробку передач (на самосвалах грузоподъемностью до 100 т) или от системы дизель-электрического привода с мотор-колесами, оснащенными индивидуальной силовой установкой - электродвигателем $[1,2]$.
Одноименнополюсные синхронные электрические машины (OCM, англ. «synchronous homopolar machines») с обмоткой возбуждения на статоре, известные также как индукторные синхронные машины и вентильно-индукторные машины независимого возбуждения, используются в ряде приложений, таких как генераторы летающих и наземных транспортных средств, сварочные генераторы и маховичные накопители энергии [3-6]. Они могут применяться в условиях высоких температур и в опасных средах как приводы [7] и как ветрогенераторы большой мощности [8]. Основными преимуществами ОСМ являются простота конструкции зубчатого ротора и высокая надежность машины в целом за счет отсутствия на роторе обмотки возбуждения или короткозамкнутой литой/сварной обмотки. Из-за недостатков асинхронных двигателей, обычно используемых в тяговых приложениях, таких как низкая надежность сварной 
обмотки ротора [9], высокие потери в роторе, трудности управления без датчика положения и сложность использования электрических тормозов при нулевой скорости вращения из-за циклического изменения теплового состояния полупроводниковых устройств инвертора, ряд исследований предлагает использовать ОСМ в качестве тягового двигателя $[5,10]$. Однако сложная трехмерная конструкция магнитопровода вызывает трудности при использовании традиционных двухмерных моделей на основе метода конечных элементов (МКЭ) для оценки характеристик ОСМ. По этой причине для ОСМ был предложен ряд оригинальных методов расчета, включая трехмерные МКЭ-модели [11, 12], двухмерные МКЭ-модели $[13,14]$, одномерные магнитные цепи и их различные комбинации $[15,16]$. В [5] предложен метод математической оптимизации тягового одноименнополюсного синхронного двигателя (ОСД). Было показано, что с помощью оптимизации можно значительно снизить потери и пульсации крутящего момента ОСД. Однако в [5] в результате оптимизации ток обмотки якоря не был уменьшен достаточно, чтобы позволить снижение номинальной мощности силовых транзисторных модулей и стоимости тягового инвертора.

В данном исследовании, в отличие от [5], выбрана целевая функция оптимизации, позволяющая значительного уменьшить ток обмотки якоря и, как след- ствие, использовать более дешевые IGBT-модули (650 A), по сравнению с модулями, используемыми в инверторе неоптимизированного ОСД (1000 А). При оптимизации учитываются ограничения, накладываемые стандартными сечениями прямоугольных обмоточных проводов. Другой целью оптимизации является снижение полных потерь мощности в двигателе.

\section{Особенности конструкции ОСД и тягового инвертора}

На рис. 1 представлены эскизы девятифазного тягового ОСД. Двигатель имеет три пары пакетов статора и ротора. На статоре размещена девятифазная шестиполюсная обмотка якоря. Обмотка возбуждения состоит из двух катушек, размещенных между пакетами статора. Статор имеет 54 паза. Ротор не имеет обмоток. Каждый пакет ротора имеет 6 зубцов, зубцы соседних пакетов ротора смещены на 30 механических градусов (180 электрических). На рис. 2, a показана зависимость максимального крутящего момента от частоты вращения электропривода карьерного самосвала БелАЗ-75570 (90 т). На рис. 2, б представлена схема девятифазного тягового инвертора ОСД. Тяговый инвертор ОСД состоит из трех отдельных трехмостовых инверторов и однофазного чоппера для питания обмотки возбуждения [13]. Более подробное описание девятифазного тягового ОСД и инвертора приведено в $[5,13]$
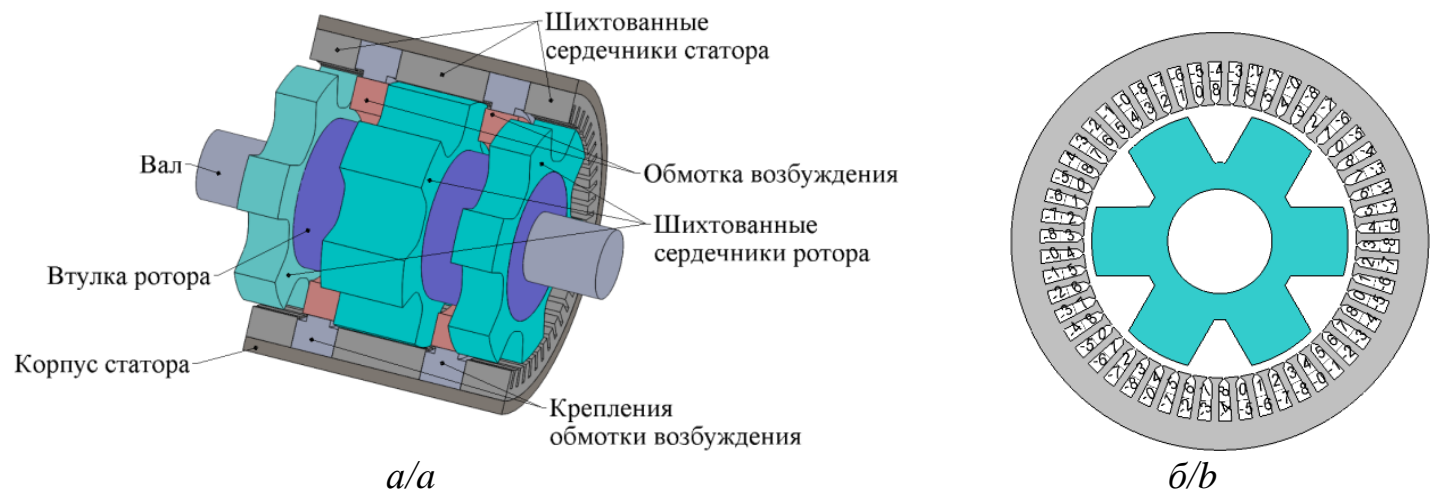

Рис. 1. Особенности конструкиии одноименнополюсного синхронного двигателя. а) общий вид двигателя. Обмотка на статоре не показана, чтобы не загромождать рисунок; б) поперечное сечение двигателя

Fig. 1. Synchronous homopolar motor design features. a) general view. The armature winding on the stator is not depicted so as not to obstruct; b) cross section

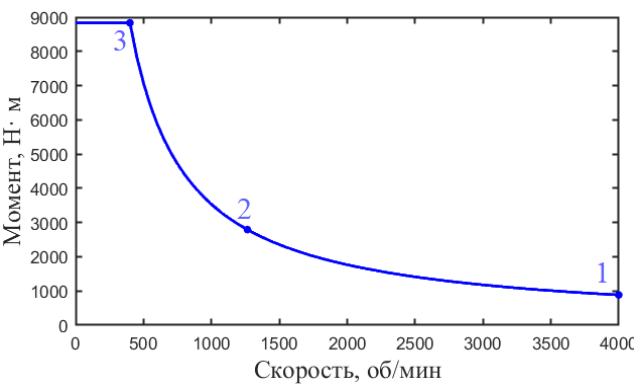

$a / a$

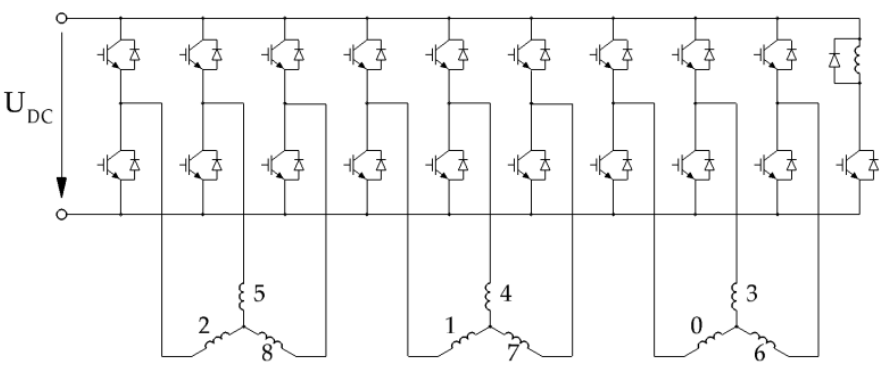

$6 / b$

Pис. 2. Диаграммы: а) тяговая характеристика двигателя карьерного самосвала; б) схема инвертора

Fig. 2. Drive diagrams: a) traction characteristic of the motor; b) inverter schematic 


\section{Целевая функция для оптимизации тягового ОСД}

Рис. 2, а показывает требуемую тяговую характеристику привода заднего колеса рассматриваемого карьерного самосвала [13]. Тяговый привод должен обеспечивать максимальный крутящий момент 8833 Н·м в диапазоне от нулевой до номинальной скорости вращения 400 об/мин. В диапазоне скорости 400 об/мин и выше привод должен обеспечивать мощность не менее 370 кВт. Максимальная скорость вращения, при которой необходимо сохранять механическую мощность 370 кВт, составляет 4000 об/мин.

В табл. 1 показаны рабочие точки двигателя, учитываемые в процедуре оптимизации: максимальный крутящий момент при номинальной скорости; номинальная мощность при максимальной скорости и промежуточная точка со средними геометрическими значениями крутящего момента и скорости. В [5] при оптимизации тягового ОСД удалось значительно снизить потери и пульсации крутящего момента, по сравнению с неоптимизированной конструкцией [13]. Также напряжение питания двигателя уменьшилось с 940 до 772 В. Однако максимальный ток якоря снизился лишь незначительно (с 886 до 816 А). По этой причине как для неоптимизированного ОСД [13], так и для оптимизированного ОСД необходимо использовать инвертор с IGBT модулями FF1000R17IE4 [17] с номинальным током 1000 А. Поскольку при наличии нескольких критериев оптимизации необходимо определить значимость отдельных критериев [18], то для значительного снижения тока якоря необходимо применение оптимизационной функции, при которой снижение тока якоря будет являться наиболее значимой целью оптимизации.

Таблица 1. Рабочие точки двигателя, учитываемые в прочедуре оптимизачии

Table 1. Operating points of the motor taken into account in optimization

\begin{tabular}{|c|c|c|c|}
\hline $\begin{array}{c}\text { Номер рабочей } \\
\text { точки } \\
\begin{array}{c}\text { Operating point } \\
\text { number }\end{array}\end{array}$ & $\begin{array}{c}\text { Момент, } \\
\mathrm{H} \cdot \mathrm{m} \\
\text { Torque, } \\
\mathrm{N} \cdot \mathrm{m}\end{array}$ & $\begin{array}{c}\text { Скорость враще- } \\
\text { ния, об/мин } \\
\text { Rotational speed, } \\
\mathrm{rpm}\end{array}$ & $\begin{array}{c}\text { Механическая } \\
\text { мощность, кВт } \\
\text { Mechanical power, } \\
\mathrm{kW}\end{array}$ \\
\hline 1 & 883 & 4000 & 370 \\
\hline 2 & 2793 & 1265 & 370 \\
\hline 3 & 8833 & 400 & 370 \\
\hline
\end{tabular}

При оптимизации тягового ОСД в данном исследовании были поставлены следующие основные цели: 1) существенно снизить ток обмотки якоря; это позволит перейти на более дешевые силовые IGBT модули FF650R17IE4 [19] в инверторе; 2) амплитуда напряжения не должна превышать 1000 B; 3) необходимо снизить общие потери двигателя, по сравнению с неоптимизированной конструкцией ОСД [5].

При оценке потерь двигателя учитываются диапазоны 1-2 и 2-3 на кривой крутящего момента двигателя (рис. 2, a). Предполагается, что средние потери в точках, в пределах диапазона, равны среднему арифметическому потерь на его границах (точки 1 и 2; точки 2 и 3 , соответственно) и что двигатель с равной вероятностью будет работать в диапазонах между точками 1-2 и между точками 2-3. По этой причине в качестве первой цели оптимизации была выбрана средняя арифметическая величина потерь в диапазонах 1-2 и 2-3, уравнение (1):

$$
\left\langle P_{\text {losses }}\right\rangle=\left(P_{\text {losses } 1}+2 \cdot P_{\text {losses } 2}+P_{\text {losses } 3}\right) / 4 \text {. }
$$

$\mathrm{B}$ качестве другой цели оптимизации выбраны пульсации момента двигателя. Рассматриваются как несимметризированные (созданные одной комбинацией пакетов ротора и статора) $T R$, так и симметризированные (созданные целым двигателем, состоящим из трех комбинаций пакетов статора и ротора) $T R_{\text {sym }}$ пульсации момента [5]. Последняя цель оптимизации - это максимальный ток обмотки якоря $I_{3}$, который достигается при максимальном крутящем моменте (рабочая точка 3).

Однокритериальный метод безусловной оптимизации Нелдера-Мида применяется в данной работе для оптимизации конструкции ОСД. Важным преимуществом метода Нелдера-Мида перед другими методами, которые часто используются для оптимизации электрических машин [20, 21], является значительное снижение времени вычислений, что позволяет увеличить количество параметров для оптимизации, а также применить более сложные критерии оптимизации, требующие расчета нескольких нагрузочных точек машины. Это преимущество важно для оптимизации тяговых машин с широким диапазоном регулирования скорости. При использовании метода Нелдера-Мида для многокритериальной оптимизации одним из возможных подходов является применение функции оптимизации в виде суммы отдельных слагаемых, каждое из которых соответствует одному из выбранных критериев оптимизации и имеет определенный весовой коэффициент, отражающий важность данного критерия оптимизации.

С учетом всех поставленных задач функция оптимизации двигателя приобретает форму:

$$
\begin{gathered}
F_{0}=\ln \left(\left\langle P_{\text {losses }}\right\rangle\right)+0,7 \cdot \ln \left(I_{3}\right)+0,05 \cdot \ln \left[\max \left(T R_{\text {sym }}\right)\right]+ \\
+0,025 \cdot \ln [\max (T R)] .
\end{gathered}
$$

Согласно функции оптимизации (2), $\left\langle P_{\text {losses }}>\right.$ являются наиболее важной целью. Вторая по важности цель - это $I_{3}$. Снижение $I_{3}$ на $1 \%$ так же ценно, как уменьшение $<P_{\text {losses }}>$ на $0,7 \%$. Уменьшение $\max \left(T R_{\text {sym }}\right)$ и $\max (T R)$ не так существенно. Уменьшение $\max \left(T R_{\text {sym }}\right)$ и $\max (T R)$ на $1 \%$ так же важно, как и уменьшение $\left\langle P_{\text {losses }}>\right.$ на $0,05 \%$ и $0,025 \%$ соответственно. Однако включение слагаемых с $\max \left(T R_{s y m}\right)$ и $\max (T R)$ позволяет ограничить увеличение пульсаций крутящего момента двигателя во время оптимизации. Весовые коэффициенты «1», «0,7», «0,05» и «0,025» не рассчитывались каким-либо формальным методом. Эти значения просто указывают приблизительную относительную важность каждой из целей оптимизации, согласно опыту авторов, при проектировании аналогичных машин.

В процедуре оптимизации также учитываются следующие ограничения:

$$
U_{D C 1}<1000 \mathrm{~B} ; B_{3}<1,65 \text { Тл; } P_{e x c}<12000 \text { Вт, }
$$


где $U_{D C 1}$ - это максимальное линейное амплитудное напряжение, достигаемое на максимально скорости (рабочая точка 1); $P_{\text {exc }}$ - это потери мощности в обмотке возбуждения и $B_{3}$ - это максимальная величина магнитной индукции в нешихтованных участках магнитопровода (втулка ротора и корпус двигателя).

Согласно поставленной задаче, необходимо также ограничить диапазон значений, которые может принимать функция оптимизации. Существуют различные способы реализации ограничений при использовании метода Нелдера-Мида. Например, целевая функция может принимать бесконечное значение, когда ограничения не выполняются. Однако такой подход приводит к быстрому уменьшению объема симплекса. Кроме того, ограничения (3) должны соблюдаться в начальном приближении и во время оптимизации. Чтобы избежать этих трудностей, к целевой функции (2) применяются «мягкие ограничения». Соответствующие слагаемые начинают быстро увеличиваться, если ограничения не выполняются. При применении «мягких ограничений» к целевой функции (2) итоговая функция оптимизации принимает вид:

$$
\begin{aligned}
& F=F_{0}+k_{1} \cdot f \cdot\left(U_{D C l} / 1000[\mathrm{~B}]-1\right)+k_{2} \cdot f \cdot\left(B_{3} / 1,65[\text { Тл }]-\right. \\
& 1)+k_{3} \cdot f \cdot\left(P_{\text {exc }} / 12000[\mathrm{BT}]-1\right), \\
& \text { где } f(x)=\left\{\begin{array}{l}
x, x>0 ; \\
0 .
\end{array}\right.
\end{aligned}
$$

В (4) первое слагаемое в правой части соответствует выражению (2), второе, третье и четвертое слагаемые реализуют ограничения (3). Отметим, что целевая функция (4) позволяет появление в процессе оптимизации вариантов конструкции, для которых ограничения не выполняются, чтобы предотвратить быстрое уменьшение объема симплекса. Однако, если коэффициенты $k_{1}, k_{2}$ и $k_{3}$ достаточно велики, оптимизированная конструкция все же будет удовлетворять ограничениям (3), поскольку при нарушении ограничений добавочные члены дают большой штрафующий вклад в величину $F$. Благодаря использованию целевой функции (4) начальное приближение может не соответствовать ограничениям (3), что и было использовано при выборе начального приближения в рассматриваемом случае. В данном исследовании предполагается, что $k_{1}=k_{2}=k_{3}=1,5$, что оказалось достаточным для того, чтобы в результате оптимизации условия (3) были выполнены.

\section{Исходные параметры конструкции ОСД и параметры, варьируемые в ходе оптимизации}

На рис. 3 представлены основные геометрические параметры тягового ОСД [13]. Параметры, которые неизменны и варьируются в ходе оптимизации, показаны в табл. 2, 3, соответственно. Внешние размеры двигателя (длина двигателя без лобовых частей обмотки $L=545$ мм и внешний радиус корпуса статора $R_{\text {housing }}=367$ мм) при оптимизации не менялись. Толщина ярма ротора и высота пакета статора также не менялись. Хотя внешний радиус корпуса статора $R_{\text {housing }}$ остается постоянным, внутренний радиус пакета статора может варьироваться при изменении толщины корпуса статора $h$. Внешний радиус ротора также зависит от ширины воздушного зазора $\delta$. Для обеспечения равных условий протекания магнитного потока возбуждения в осевом направлении через корпус статора и втулку ротора площади их поперечных сечений задаются равными. В результате внешний радиус втулки ротора определяется по формуле:

$$
R_{\text {sleeve }}=\sqrt{ }\left(R_{\text {shaft }}^{2}+R_{\text {housing }}^{2}-\left[R_{\text {housing }}-h\right]^{2}\right) .
$$

Для неоптимизированной конструкции, описанной в [13], $R_{\text {sleeve }}=161$ мм, в то время как расчет по формуле (5) дает $R_{\text {sleeve }}=167$ мм. Поскольку толщина ярма ротора, равная $R_{1}-R_{\text {sleeve, }}$ не меняется, глубина паза ротора изменяется не только с изменением внешнего диаметра ротора, но и с изменением $R_{\text {sleeve }}$.

Изменение угловых размеров между зубцами ротора на внутреннем и внешнем радиусе ротора осуществлялось согласованно путем умножения обоих размеров на коэффициент $f_{r s}$. Продольный и радиальный размеры, занимаемые обмоткой возбуждения между двумя пакетами статора, составляют $L_{e x}=43$ мм и $h_{\text {exc }}=78 \mathrm{мм}$, соответственно [5]. Во время оптимизации $L_{e x}=\left(L-L_{\text {stat }}\right) / 2-\Delta_{\text {a }}$ изменялось совместно с $L_{\text {stat }}$. Размер $h_{\text {exc }}=R_{\text {housing }}-h-h_{\text {lam }}-R_{\text {sleeve }}-\Delta_{\mathrm{r}}$ изменяется путем изменения $h$, а также с изменением радиуса $R_{\text {sleeve }}$, который, согласно (5), также является функцией $h$.
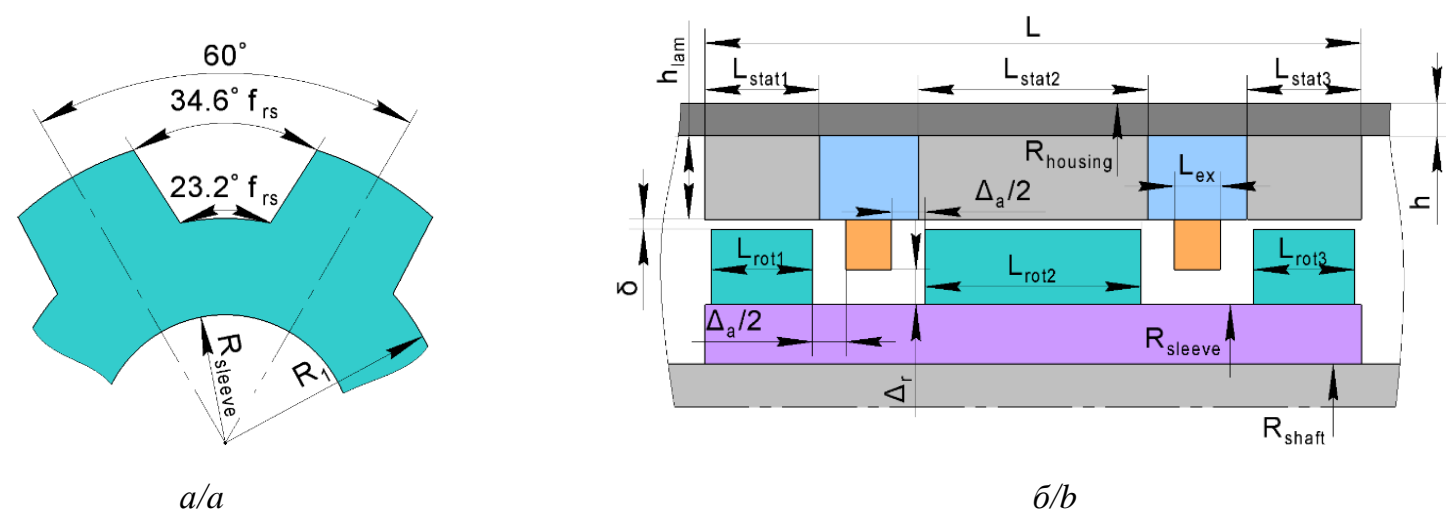

$\sigma / b$

Рис. 3. Геометрические параметры двигателя: а) размеры ротора; б) прочие размеры статора и ротора

Fig. 3. Geometric parameters of the motor: a) rotor radial dimensions; $b$ ) other stator and rotor dimensions 
Таблица 2. Некоторые геометрические параметры двигателя, неизменяемые в ходе оптимизацчии

Table 2. Some geometric parameters of the motor invariable during the optimization

\begin{tabular}{|c|c|}
\hline Параметр/Parameter & $\begin{array}{c}\text { Величина } \\
\quad[13] \\
\text { Value [13] }\end{array}$ \\
\hline $\begin{array}{l}\text { Длина машины без лобовых частей обмотки } L, \text { мм } \\
\text { Machine length without end winding parts } L, \mathrm{~mm}\end{array}$ & 545 \\
\hline $\begin{array}{l}\text { Длины пакетов статора } L_{\text {stat } 1} ; L_{\text {stat }} ; L_{\text {stat } 3}, \mathrm{Mм} \\
\text { Lengths of the stator stacks } L_{\text {stat } 1} ; L_{\text {stat } 2} ; L_{\text {stat } 3}, \mathrm{~mm}\end{array}$ & $\begin{array}{c}101 ; 197 \\
101\end{array}$ \\
\hline $\begin{array}{l}\text { Длины пакетов ротора } L_{\text {rot } 1} ; L_{\text {rot } 2} ; L_{\text {rot } 3}, \text { мм } \\
\text { Lengths of the rotor stacks } L_{\text {rot } 1} ; L_{r o t 2} ; L_{r o t 3}, \mathrm{~mm}\end{array}$ & $\begin{array}{l}92 ; 184 \\
92\end{array}$ \\
\hline $\begin{array}{l}\text { Осевой зазор между обмоткой возбуждения и рото- } \\
\text { poм } \Delta_{\mathrm{a}} \text {, мм } \\
\text { Axial clearance between field winding and rotor } \Delta_{a}, \mathrm{~mm}\end{array}$ & 30 \\
\hline $\begin{array}{l}\text { Радиальный зазор между обмоткой возбуждения и } \\
\text { ротором } \Delta_{\mathrm{r}} \text {, мм } \\
\text { Radial clearance between field winding and rotor } \Delta_{r}, \mathrm{~mm}\end{array}$ & 27 \\
\hline $\begin{array}{l}\text { Толщина ярма ротора } R_{1}-R_{\text {sleeve }}, \mathrm{Mm} \\
\text { Rotor yoke thickness } R_{1}-R_{\text {sleeve }}, \mathrm{mm}\end{array}$ & 22,8 \\
\hline $\begin{array}{l}\text { Радиус вала } R_{\text {shaff }}, \text { мм } \\
\text { Shaft radius } R_{\text {shaff }}, \mathrm{mm}\end{array}$ & 70 \\
\hline $\begin{array}{l}\text { Высота пакета статора } h_{\text {lam }}, \text { мм } \\
\text { Stator lamination height } h_{\text {lam }}, \mathrm{mm}\end{array}$ & 65 \\
\hline $\begin{array}{l}\text { Внешний радиус корпуса двигателя } R_{\text {housing }}, \text { мм } \\
\text { External radius of the motor housing } R_{\text {housing }}, \mathrm{mm}\end{array}$ & 367 \\
\hline
\end{tabular}

Таблица 3. Параметры двигателя, варьируемые в ходе оптимизации

Table 3. $\quad$ Parameters of the motor varied during the optimization

\begin{tabular}{|c|c|}
\hline Параметр/Parameter & $\begin{array}{l}\text { Исходная величина пе- } \\
\text { ред оптимизацией [13] } \\
\text { Initial value before } \\
\text { the optimization [13] } \\
\end{array}$ \\
\hline $\begin{array}{l}\text { Толщина корпуса } h, \text { мм } \\
\text { Housing thickness } h, \mathrm{~mm} \\
\end{array}$ & 36 \\
\hline $\begin{array}{l}\text { Полная длина пакетов статора } L_{\text {stator }}, \text { мм } \\
\text { Total stator stacks length } L_{\text {stator }}, \mathrm{mm}\end{array}$ & 399 \\
\hline $\begin{array}{l}\text { Величина воздушного зазора } \delta, \text { мм } \\
\text { Airgap width } \delta, \mathrm{mm}\end{array}$ & 2,3 \\
\hline $\begin{array}{l}\text { Коэффициент паза ротора } f_{r s} \\
\text { Rotor slot factor } f_{r s}\end{array}$ & 1 \\
\hline $\begin{array}{l}\text { Углы ослабления поля в рабочих точ- } \\
\text { ках } 1,2,3 \text {, электрические радианы } \\
\text { Angles of field weakening at operating } \\
\text { points } 1,2,3 \text {, electrical radians }\end{array}$ & 0,$61 ; 0,3 ; 0,25$ \\
\hline $\begin{array}{l}\text { Плотность потока магнитного монопо- } \\
\text { ля в рабочих точках } 1,2,3, \text { Вб/м } \\
\text { Magnetic monopole densities at operating } \\
\text { points } 1,2,3, \mathrm{~Wb} / \mathrm{m}\end{array}$ & 0,$48 ; 0,63 ; 1,2$ \\
\hline
\end{tabular}

В [13] было принято, что сопротивление обмотки возбуждения равно 10,2 Ом. В данном исследовании сопротивление обмотки возбуждения в зависимости от размеров обмотки возбуждения меняется в ходе оптимизации и составляет 10,2 Ом.[43 мм].[78 мм $] / h_{e x d} / L_{e x}$. Число витков обмотки возбуждения полагается неизменным и равным 340. Табл. 4 показывает характеристики обмотки якоря.

Для лучшего использования питающего напряжения количество витков обмотки якоря было увеличено, в сравнении с [13], что привело к увеличению высоты паза статора и уменьшению толщины шихтованного ярма статора. Для ограничения увеличения размера паза статора было выбрано меньшее сечение проводников обмотки. Основные характеристики прототипа двигателя, описанного в [13], показаны в левой части табл. 5. Основные характеристики двигателя, полученные после вышеуказанных изменений (начальное приближение для оптимизации), показаны в правой части табл. 5.

По сравнению с конструкцией, описанной в [5], согласно формуле (5), падение магнитодвижущей силы обмотки возбуждения (МДС) на втулке ротора уменьшается за счет увеличения $R_{\text {sleeve }}$. роны, уменьшаются глубина пазов и явнополюсность ротора. Меняется также сопротивление обмотки возбуждения. Кроме того, параметры обмотки якоря для конструкции, используемой в качестве начального приближения для оптимизации, также отличаются от [5], что показано в табл. 4. Как видно из табл. 5, изменение параметров обмотки якоря, показанных в табл. 4, привело к значительному увеличению линейного напряжения до 1303 В для первой рабочей точки. Это значение напряжения значительно превышает максимально допустимое напряжение в цепи постоянного тока инвертора карьерного самосвала и не может быть реализовано на практике. Однако в следующем разделе будет показано, что с помощью целевой функции (4) и метода Нелдера-Мида можно значительно улучшить все основные характеристики ОСД, без превышения предела напряжения 1000 B.

\section{Оптимизация ОСД с помощью метода Нелдера-Мида}

В процессе оптимизации ОСД использовались традиционный алгоритм Нелдера-Мида [22], математическая модель ОСД на основе двухмерной МКЭмодели, описанная в [13], и целевая функция (4). Процедура оптимизации варьирует 10 параметров ОСД, приведенных в табл. 2. На рис. 4 показано поперечное сечение двигателя и амплитуда магнитной индукции до и после оптимизации в рабочей точке 3 (табл. 1) с максимальным крутящим моментом, что соответствует наиболее насыщенному состоянию магнитопровода; области поперечного сечения с экстремальным уровнем насыщения более 2 Тл выделены черными контурами. Видно, что после оптимизации площадь областей с экстремальным насыщением уменьшилась. В табл. 6 показаны величины варьируемых конструктивных параметров ОСД после оптимизации. На рис. 5 показано изменение в ходе оптимизации таких значений, как общие потери $\left\langle P_{\text {losses }}>\right.$, амплитуда тока якоря в рабочей точке $3 I_{3}$, амплитуда линейного напряжения в рабочей точке 1 и значение целевой функции $F(4)$. В табл. 7 сравниваются основные характеристики двигателя до и после оптимизации. Как видно из табл. 6, в рабочих точках 1, 2 и 3 (работа в двигательном режиме) общие потери после оптимизации снижены в 1,09, 1,19 и 1,04 paза, соответственно. После оптимизации пульсации крутящего момента лишь немного уменьшились в рабочей точке 1 . Напряжение в рабочей точке 1 уменьшилось в 1,3 раза, поэтому максимальная амплитуда напряжения составляет $988 \mathrm{~B}$, что не превышает 
ограничения в 1000 В (3). Также в рабочей точке 3 амплитудное значение тока обмотки якоря уменьшилось с 669 до 601 А. Хотя в начальном приближении из-за увеличения количества витков амплитудное значение тока обмотки якоря намного меньше, чем для серийной машины [13], однако начальное приближение не проходит по ограничению напряжения (3) и, следовательно, не может быть использовано на практике. Использование оптимизации позволило не только снизить линейное напряжение в рабочей точке 1 до допустимого уровня, но и дополнительно снизить ток обмотки якоря.

Поскольку максимальное значение амплитуды тока якоря для оптимизированного ОСД не превышает 650 А, то в инверторе можно использовать IGBT модули FF650R17IE4 вместо модулей FF1000R17IE4, которые применяются в серийно выпускаемом тяговом приводе с ОСД [13]. Стоимость модуля FF1000R17IE4 составляет 840 долларов США, а стоимость модуля FF650R17IE4 в 1,4 раза меньше и составляет 585 долларов США. Цены указаны на сайте производителя IGBT модулей [23]. Таким образом, для девятифазного инвертора экономия только на IGBT модулях составляет (840-585)·9=2295 долларов США. Кроме того, составляющая переменного тока в конденсаторной батарее звена постоянного тока уменьшится в 1,4 раза, что позволит снизить емкость конденсаторной батареи звена постоянного тока, что также приведет к снижению стоимости инвертора.

Таблица 4. Параметры обмотки якоря, неизменяемые в ходе оптимизаиии

Table 4. Winding parameters invariable during the optimization

\begin{tabular}{|c|c|c|}
\hline Параметр/Parameter & $\begin{array}{c}\text { Параметры } \\
\text { прототипа ОСД, } \\
\text { описанного в } \\
\text { [13] } \\
\text { SHM prototype } \\
\text { described in [13] }\end{array}$ & $\begin{array}{c}\text { Начальное } \\
\text { приближение } \\
\text { для оптими- } \\
\text { зации } \\
\text { New initial } \\
\text { design } \\
\end{array}$ \\
\hline $\begin{array}{l}\text { Число витков в слое } \\
\text { Number of turns per stator ar- } \\
\text { mature layer }\end{array}$ & 5 & 7 \\
\hline $\begin{array}{l}\text { Число параллельных ветвей в } \\
\text { витке } \\
\text { Number of parallel strands per } \\
\text { turn of the stator armature coil }\end{array}$ & 2 & 2 \\
\hline $\begin{array}{l}\text { Размеры прямоугольного } \\
\text { провода, мм² } \\
\text { Dimensions of armature wire } \\
\text { winding, } \mathrm{mm}^{2}\end{array}$ & $3,15 \cdot 4,5$ & $2,5 \cdot 4,5$ \\
\hline $\begin{array}{l}\text { Высота части паза статора, } \\
\text { заполненной обмоткой, мм } \\
\text { Height of the stator slot part } \\
\text { filled with the wire, } \mathrm{mm}\end{array}$ & 36,4 & 41,1 \\
\hline $\begin{array}{l}\text { Сопротивление обмотки воз- } \\
\text { буждения, Ом } \\
\text { Excitation winding resistance, } \\
\text { Ohm }\end{array}$ & 10,2 & 16,8 \\
\hline
\end{tabular}

Таблица 5. Сравнение характеристик прототипа двигателя, описанного в [13], и начального приближения, используемого для оптимизаиии

Table 5. Comparison of the characteristics of the motor prototype described in [13] and the characteristics of the new initial design used as the starting point for optimization

\begin{tabular}{|c|c|c|c|c|c|c|c|c|}
\hline \multirow{2}{*}{$\begin{array}{l}\text { Величина/Value } \\
\text { Рабочая точка } \\
\text { Operating point } \\
\end{array}$} & \multicolumn{4}{|c|}{$\begin{array}{l}\text { Прототип ОСД, описанный в [13] } \\
\text { SHM prototype described in [13] }\end{array}$} & \multicolumn{4}{|c|}{$\begin{array}{l}\text { Начальное приближение для оптимизации } \\
\text { New initial design (before the optimization) }\end{array}$} \\
\hline & 1 & 2 & 3 & $\begin{array}{c}\text { Тормозной режим } \\
\text { Brake mode }\end{array}$ & 1 & 2 & 3 & $\begin{array}{l}\text { Tормозной режим } \\
\text { Brake mode }\end{array}$ \\
\hline $\begin{array}{l}\text { Скорость вращения, об/мин } \\
\text { Rotational speed, rpm }\end{array}$ & 4000 & 1265 & 400 & 1100 & 4000 & 1265 & 400 & 1100 \\
\hline $\begin{array}{l}\text { Амплитуда тока, А } \\
\text { Current, A ampl }\end{array}$ & 197 & 408 & 886 & 643 & 142 & 296 & 669 & 485 \\
\hline $\begin{array}{l}\text { Механическая мощность, кВт } \\
\text { Mechanical power, } \mathrm{kW}\end{array}$ & 370 & 370 & 370 & -540 & 370 & 370 & 370 & -540 \\
\hline $\begin{array}{l}\text { Активная мощность, кВт } \\
\text { Active power, } \mathrm{kW}\end{array}$ & 412 & 387 & 404 & -508 & 412 & 387 & 405 & -509 \\
\hline $\begin{array}{l}\text { КПД, \% } \\
\text { Efficiency, \% }\end{array}$ & 89,8 & 95,4 & 90,0 & 93,8 & 89,8 & 95,3 & 89,8 & 94,0 \\
\hline $\begin{array}{l}\text { Полные потери, кBт } \\
\text { Total losses, kW }\end{array}$ & 41,9 & 18,0 & 41,0 & 32,2 & 42,2 & 18,1 & 42,1 & 32,4 \\
\hline $\begin{array}{l}\text { Коэффициент мощности } \\
\text { Power factor }\end{array}$ & 0,99 & 0,82 & 0,91 & $-0,65$ & 0,99 & 0,81 & 0,88 & $-0,62$ \\
\hline $\begin{array}{l}\text { Амплитуда линейного напряжения, В } \\
\text { Line voltage, V ampl }\end{array}$ & 940 & 472 & 196 & 462 & 1303 & 661 & 272 & 642 \\
\hline $\begin{array}{l}\text { Несимметризированные пульсации момента, Н·м } \\
\text { Not symmetrized torque ripple, } \mathrm{N} \cdot \mathrm{m}\end{array}$ & 71,9 & 61,5 & 24,1 & 42,1 & 71,9 & 62,1 & 24,1 & 43,5 \\
\hline $\begin{array}{l}\text { Симметризированные пульсации момента, } \mathrm{H} \cdot \mathrm{M} \\
\text { Symmetrized torque ripple, } \mathrm{N} \cdot \mathrm{m}\end{array}$ & 21,0 & 12,4 & 2,8 & 8,4 & 20,8 & 12,3 & 2,6 & 8,1 \\
\hline $\begin{array}{l}\text { Excitation current, A } \\
\text { Ток возбуждения, A }\end{array}$ & 5,6 & 8,1 & 26,3 & 10,7 & 5,5 & 8,1 & 24,8 & 10,8 \\
\hline $\begin{array}{l}\text { Магнитная индукция в нешихтованных частях } \\
\text { магнитопровода, Тл } \\
\text { Flux density in non-laminated parts of the magnetic } \\
\text { core, T }\end{array}$ & 0,59 & 0,77 & 1,46 & 0,77 & 0,59 & 1,04 & 1,65 & 0,98 \\
\hline
\end{tabular}


Таблица 6. Величины варьируемых параметров двигателя после оптимизации

Table 6. $\quad$ Varied design parameters of the traction motor after optimization

\begin{tabular}{|c|c|}
\hline Parameter/Параметр & $\begin{array}{c}\text { Optimal design } \\
\text { Оптимальная конструкция }\end{array}$ \\
\hline $\begin{array}{l}\text { Толщина корпуса } h, \text { мм } \\
\text { Housing thickness } h, \mathrm{~mm}\end{array}$ & 32,8 \\
\hline $\begin{array}{l}\text { Полная длина пакетов статора } L_{\text {stator }}, \text { мм } \\
\text { Total stator stacks length } L_{\text {stator }}, \mathrm{mm}\end{array}$ & 431 \\
\hline $\begin{array}{l}\text { Величина воздушного зазора } \delta \text {, мм } \\
\text { Airgap width } \delta, \mathrm{mm}\end{array}$ & 2,41 \\
\hline $\begin{array}{l}\text { Коэффициент паза ротора } f_{r s} \\
\text { Rotor slot factor } f_{r s}\end{array}$ & 1,10 \\
\hline $\begin{array}{l}\text { Углы ослабления поля в рабочих точках 1, 2, 3, электрические радианы } \\
\text { Angles of field weakening at operating points 1, 2, 3, electrical radians }\end{array}$ & 0,$762 ; 0,400 ; 0,364$ \\
\hline $\begin{array}{l}\text { Плотность потока магнитного монополя в рабочих точках 1, 2, 3, Вб/м } \\
\text { Magnetic monopole densities at operating points } 1,2,3, \mathrm{~Wb} / \mathrm{m}\end{array}$ & 0,$331 ; 0,678 ; 1,139$ \\
\hline
\end{tabular}

Таблица 7. Результаты оптимизащии

Table 7. Optimization results

\begin{tabular}{|c|c|c|c|c|c|c|c|c|}
\hline $\begin{array}{l}\text { Величина } \\
\text { Value }\end{array}$ & Началь & $\begin{array}{r}\text { Hoe пр } \\
\mathrm{N} \\
\text { (befo }\end{array}$ & $\begin{array}{l}\text { чближс } \\
\text { ew init } \\
\text { e the o }\end{array}$ & $\begin{array}{l}\text { ние до оптимизации } \\
\text { al design } \\
\text { otimization) }\end{array}$ & & & $\begin{array}{l}\text { ле опт } \\
\text { ter opt }\end{array}$ & $\begin{array}{l}\text { имизации } \\
\text { mization }\end{array}$ \\
\hline $\begin{array}{l}\text { Рабочая точка } \\
\text { Operating point }\end{array}$ & 1 & 2 & 3 & $\begin{array}{l}\text { Тормозной режим } \\
\text { Brake mode }\end{array}$ & 1 & 2 & 3 & \begin{tabular}{|c|} 
Тормозной режим \\
Brake mode
\end{tabular} \\
\hline $\begin{array}{l}\text { Скорость вращения, об/мин } \\
\text { Rotational speed, rpm }\end{array}$ & 4000 & 1265 & 400 & 1100 & 4000 & 1265 & 400 & 1100 \\
\hline $\begin{array}{l}\text { Амплитуда тока якоря, А } \\
\text { Armature current, A ampl }\end{array}$ & 142 & 296 & 669 & 485 & 176 & 255 & 601 & 429 \\
\hline $\begin{array}{l}\text { Механическая мощность, кBт } \\
\text { Mechanical power, kW }\end{array}$ & 370 & 370 & 370 & -540 & 370 & 370 & 370 & -540 \\
\hline $\begin{array}{l}\text { Механические потери, кВт } \\
\text { Mechanical losses, kW }\end{array}$ & 17,57 & 0,65 & 0,05 & 0,45 & $\begin{array}{c}17,5 \\
7 \\
\end{array}$ & 0,65 & 0,05 & 0,45 \\
\hline $\begin{array}{l}\text { Омические потери в обмотке якоря, кВт } \\
\text { Conductive armature winding losses, kW }\end{array}$ & 1,4 & 6,1 & 30,9 & 16,2 & 2,1 & 4,5 & 25,0 & 12,7 \\
\hline $\begin{array}{l}\text { Потери в обмотке якоря на вихревые тока, кВт } \\
\text { Eddy-current armature winding losses, } \mathrm{kW}\end{array}$ & 5,2 & 2,5 & 1,1 & 4,3 & 6,0 & 2,1 & 1,0 & 3,8 \\
\hline $\begin{array}{l}\text { Потери в стали статора, кBт } \\
\text { Stator core losses, kW }\end{array}$ & 15,1 & 7,5 & 2,4 & 9,4 & 10,9 & 5,9 & 2,5 & 8,2 \\
\hline $\begin{array}{l}\text { Потери в стали ротора, кBт } \\
\text { Rotor core losses, kW }\end{array}$ & 2,5 & 0,6 & 0,1 & 0,6 & 1,5 & 0,6 & 0,1 & 0,5 \\
\hline $\begin{array}{l}\text { Потери в обмотке возбуждения, кВт } \\
\text { Excitation losses, kW }\end{array}$ & 0,4 & 0,8 & 7,5 & 1,4 & 0,5 & 1,5 & 11,9 & 2,0 \\
\hline $\begin{array}{l}\text { Активная мощность, кВт } \\
\text { Active power, kW }\end{array}$ & 412 & 387 & 405 & -509 & 408 & 384 & 399 & -514 \\
\hline $\begin{array}{l}\text { КПД, \% } \\
\text { Efficiency, \% }\end{array}$ & 89,8 & 95,3 & 89,8 & 94,0 & 90,5 & 96,1 & 90,1 & 94,9 \\
\hline $\begin{array}{l}\text { Полные потери (двигатель), кВт } \\
\text { Total losses (motor), kW }\end{array}$ & 42,2 & 18,1 & 42,1 & 32,4 & 38,7 & 15,2 & 40,5 & 27,6 \\
\hline $\begin{array}{l}\text { Коэффициент мощности } \\
\text { Power factor }\end{array}$ & 0,99 & 0,81 & 0,88 & $-0,62$ & 0,97 & 0,96 & 0,96 & $-0,74$ \\
\hline $\begin{array}{l}\text { Амплитуда линейного напряжения, В } \\
\text { Line voltage, V ampl }\end{array}$ & 1303 & 661 & 272 & 642 & 988 & 632 & 275 & 621 \\
\hline $\begin{array}{l}\text { Симметризированные пульсации момента, } \mathrm{H} \cdot \mathrm{m} \\
\text { Symmetrized torque ripple, } \mathrm{N} \cdot \mathrm{m}\end{array}$ & 20,8 & 12,3 & 2,6 & 8,1 & 18,9 & 12,5 & 3,0 & 9,4 \\
\hline $\begin{array}{l}\text { Ток возбуждения, А } \\
\text { Excitation current, A }\end{array}$ & 5,5 & 8,1 & 24,8 & 10,8 & 5,5 & 9,5 & 26,6 & 10,8 \\
\hline $\begin{array}{l}\text { Магнитная индукция в не шихтованных частях } \\
\text { магнитопровода, Тл } \\
\text { Flux density in non-laminated parts of the magnetic } \\
\text { core, } T\end{array}$ & 0,59 & 0,77 & 1,46 & 0,77 & 0,48 & 0,98 & 1,65 & 0,91 \\
\hline
\end{tabular}




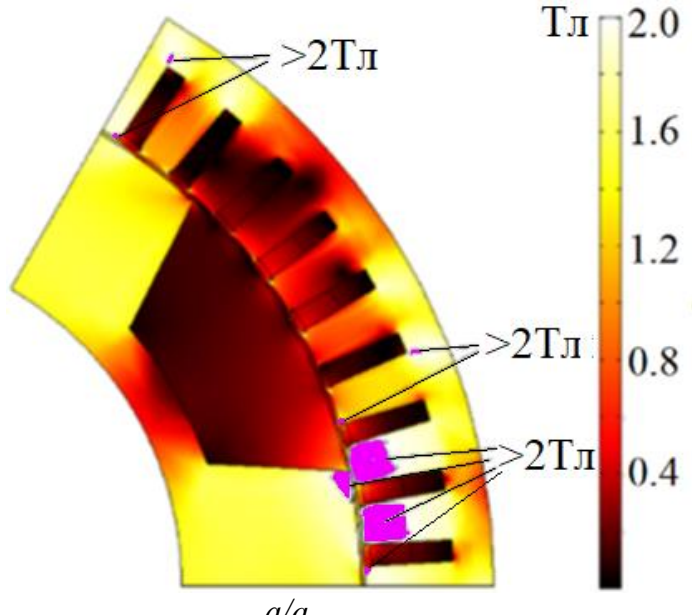

$a / a$

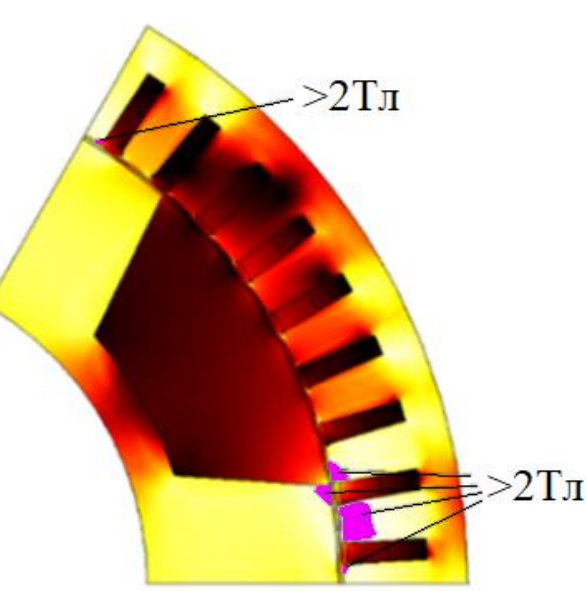

$\sigma / b$

Pис. 4. Поперечное сечение двигателя и величина магнитной индукиии; розовым иветом отмечены области с экстремальным уровнем насыщения (>2 Тл): а) до оптимизации; б) после оптимизации; видно, что после оптимизачии площадь областей с магнитной индукцией $>2$ Тл заметно уменьшилась

Fig. 4. Motor cross section and the magnitude plot of flux density; black outlines mark the extreme saturation level (>2 T): a) before optimization; b) after optimization; it can be observed that after optimization, the area of regions with flux density >2 Thas noticeably decreased

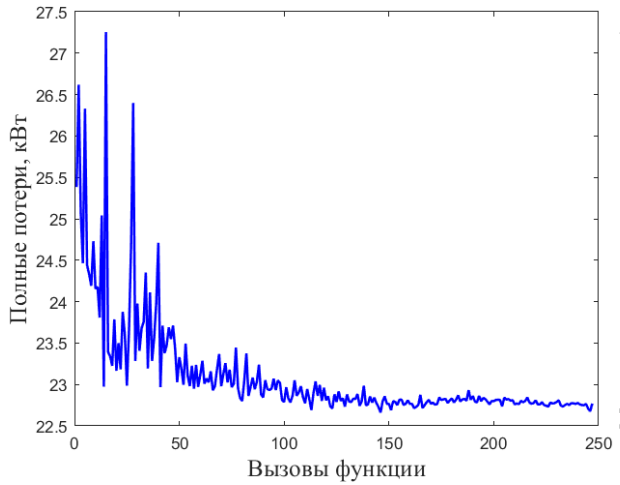

a/a

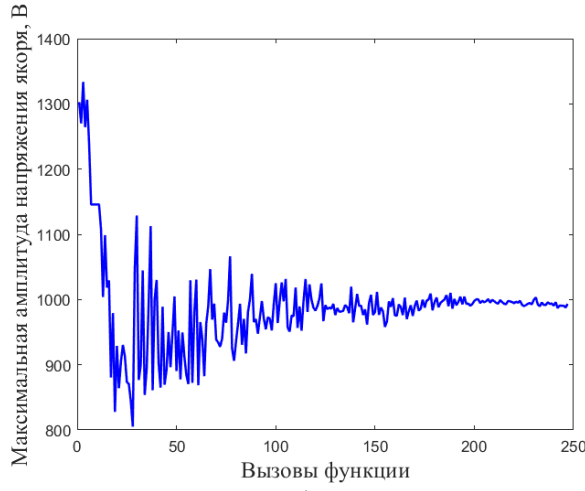

B/c

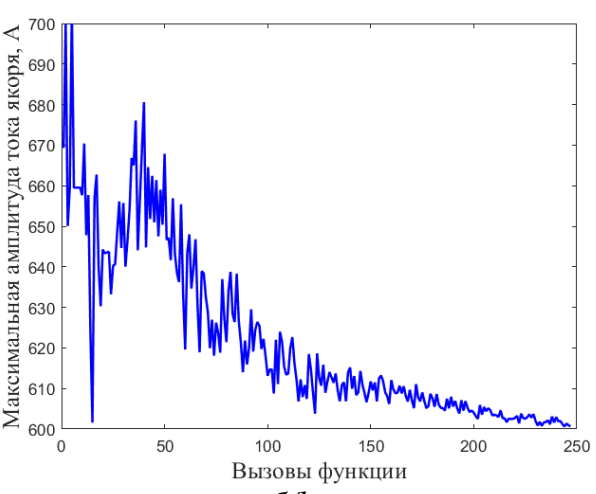

$\sigma / b$

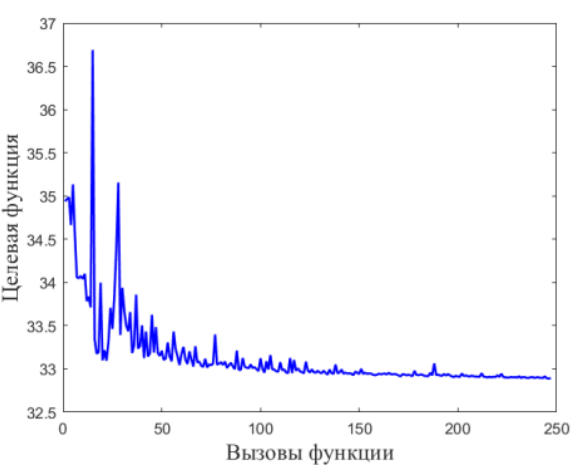

$2 / d$

Рис. 5. Изменение целевых параметров в ходе оптимизачии: а) полные потери $<P l_{\text {osses }}>$; б) максимальная амплитуда тока обмотки якоря; в) максимальная амплитуда напряжения обмотки якоря; г) целевая функиия оптимизации $F$

Fig. 5. Variations in the objectives during the optimization: a) total losses $\left\langle P l_{\text {osses }}\right\rangle$; b) maximum current amplitude in the armature winding; c) maximum voltage amplitude in the armature winding; $d$ ) objective function $F$

\section{Заключение}

Применение неэффективных методов проектирования одноименнополюсных синхронных двигателей может привести к необходимости значительно завышать установленную мощность силового инвертора в тяговых приложениях, требующих работы в широком диапазоне скоростей с постоянной мощностью. В статье обсуждается новая процедура и результаты оптимизации серийно производимого тягового одноименнополюсного синхронного двигателя с номинальной мощностью 370 кВт с использованием метода Нелдера-Мида. Целевая функция составлена для улучше- 
ния таких основных характеристик тягового одноименнополюсного синхронного двигателя, как суммарные потери мощности двигателя и максимальный ток обмотки якоря. При разработке оптимизированной конструкции, которая может быть применена на практике, накладываются необходимые ограничения. В результате оптимизации потери в двигателе и максимальный ток, потребляемый двигателем от инвер-

\section{СПИСОК ЛИТЕРАТУРЫ}

1. Трухнов Л.И. Новые и перспективные разработки ОАО «БелАЗ» - управляющая компания холдинга «БелАЗ-холдинг» горнякам // Горный информационно-аналитический бюллетень (научно-технический журнал). - 2015. - № S1-2. - С. 34-41.

2. Recent advances in mining haul trucks / W. Koellner, G. Brown, J. Rodriguez, J. Pontt, P. Cortes, H. Miranda // IEEE Transactions on Industrial Electronics. - 2004. - V. 51. - P. 321-329. DOI: 10.1109 /TIE.2004.825263

3. Superconducting AC homopolar machines for high-speed applications / S. Kalsi, K. Hamilton, R.G. Buckley, R.A. Badcock // Energies. - 2019. - V. 12. - P. 1-14. DOI: 10.3390/en12010086

4. Homopolar generators: an overview / C. Bianchini, F. Immovilli, A. Bellini, E. Lorenzani, C. Concari, M. Scolari // Proceedings of the 2011 IEEE Energy Conversion Congress and Exposition. Phoenix, USA, 17-22 September 2011. - P. 1523-1527. DOI: doi.org/10.1109/ECCE.2011.6063962

5. Design optimization of a traction synchronous homopolar motor / V. Dmitrievskii, V. Prakht, A. Anuchin, V. Kazakbaev // Mathematics. - 2021. - V. 9. - P. 1-14. DOI: 10.3390/math9121352

6. A novel critical analysis method of homopolar inductor alternator for preliminary design in capacitor charge power supply / K. Yu, J. Yao, X. Xie, P. Tang // IEEE Transactions on Plasma Science. - 2019. V. 47. - № 5. - P. 2354-2361. DOI: 10.1109/TPS.2019.2892610.

7. Dual-purpose no-voltage winding design for the bearingless AC homopolar and consequent pole motors / E. Severson, R. Nilssen, T. Undeland, N. Mohan // IEEE Transactions on Industry Applications. - 2015. - V. 51. - № 4. - P. 2884-2895. DOI: 10.1109/TIA.2015.2388852

8. Design of a 10-MW-Class HTS homopolar generator for wind turbines / J.-S Jeong, D.-K. An, J.-P. Hong, H.-J. Kim, Y.-S. Jo // IEEE Transactions on Applied Superconductivity. - 2017. V. 27. - № 4. - P. 1-4. DOI: 10.1109/TASC.2017.2669140.

9. Traction induction motor / J. Bernatt, S. Gawron, T. Glinka, A. Polak // Proc. of the 13th International Conference Modern Electrified Transport (MET). - Warsaw, Poland, 5-7 October 2017. - P. 1-5. DOI: 10.1051/matecconf/201818004005

10. Комплект тягового электрооборудования для БелАЗ-90 на базе вентильно-индукторного электродвигателя с независимым возбуждением / А.С. Анучин, М.К. Котельников, В.Н. Остриров, М.М. Лашкевич, М.В. Подлесный, К.В. Мильский, А.С. Тарасов // Труды X международной конференции по автоматизированному электроприводу АЭП. - Новочеркасск: Изд-во ООО «Лик», 2018 - С. 218-224

11. Cheshmehbeigi H., Afjei E. Design optimization of a homopolar salientpole brushless DC machine: analysis, simulation, and experimental tests // IEEE Transactions on Energy Conversion. - 2013. - V. 28. - № 2. P. 289-297. DOI: 10.1109/TEC.2013.2249584. тора, были значительно снижены. Достигнутое снижение максимального тока позволяет снизить стоимость силовых полупроводниковых модулей инвертора в 1,4 раза, а также позволяет снизить переменную составляющую тока в звене постоянного тока.

Работа выполнена при поддержке гранта Российского научного фонда (проект № 21-19-00696).

12. Outer-rotor AC homopolar motors for flywheel energy storage / E. Severson, N. Mohan, R. Nilssen, T. Undeland // Proc. of 7th IET Int. Conf. Power Electron., Mach. Drives (PEMD). - Manchester, U.K., 8-10 April 2014. - P. 1-6. DOI: 10.1049/cp.2014.0345.

13. Traction synchronous homopolar motor: simplified computation technique and experimental validation / V. Dmitrievskii, V. Prakht, A. Anuchin, V. Kazakbaev // IEEE Access. - 2020. - V. 2020. P. 185112-185120. DOI: 10.1109/ACCESS.2020.3029740

14. Investigation of a two-dimensional analytical model of the homopolar inductor alternator / J. Yang, C. Ye, X. Liang, W. Xu, F. Xiong, Y. Xiang, W. Li // IEEE Transactions on Applied Superconductivity. - 2018. - V. 28. - № 3. - P. 1-5. DOI: 10.1109/TASC.2018.2802480

15. Relationship between homopolar inductor machine and woundfield synchronous machine / C. Ye, J. Yang, F. Xiong, Z.Q. Zhu // IEEE Transactions on Industrial Electronics. - 2020. - V. 67. P. 919-930. DOI: 10.1109/TIE.2019.2898577

16. Belalahy C., Rasoanarivo I., Sargos F. Using 3D reluctance network for design a three phase synchronous homopolar machine // Proceedings of the 2008 34th Annual Conference of IEEE Industrial Electronics. - Orlando, USA, 10-13 November 2008. P. 2067-2072. DOI: 10.1109/IECON.2008.4758275

17. FF1000R17IE4, IGBT-Modules, Technical Information, Revision 3.2, Infineon. 2013. URL: https://www.infineon.com/dgdl/ Infineon-FF1000R17IE4-DS-v03_02-EN.pdf?fileId=db3a3043 1ff9881501201dc994a34980 (дата обращения 08.09.2021).

18. Prakht V., Dmitrievskii V., Kazakbaev V. Optimal design of gearless flux-switching generator with ferrite permanent magnets // Mathematics - 2020 - V. 8 - P. 1-14. DOI: 10.3390/math8020206

19. FF650R17IE4, IGBT-Modules, Technical Information, Revision 3.3, Infineon. 2013. URL: https://www.infineon.com/dgdl/ Infineon-FF650R17IE4-DS-v03_03-EN.pdf?fileId=db3a3043 $1 \mathrm{ff} 9881501201 \mathrm{dcfe} 2 \mathrm{a} 54986$ (дата обращения 08.09.2021)

20. Cupertino F., Pellegrino G., Gerada C. Design of synchronous reluctance machines with multiobjective optimization algorithms // IEEE Transactions on Industry Applications. - 2014. - V. 50. P. 3617-3627. DOI: 10.1109/TIA.2014.2312540

21. Krasopoulos C.T., Beniakar M.E., Kladas A.G. Robust optimization of high-speed PM motor design // IEEE Transactions on Magnetics. 2017. - V. 53. - P. 1-4. DOI: 10.1109/TMAG.2017.2660238

22. Nelder J.A., Mead R. A simplex method for function minimization // The Computer Journal. - 1965. - V. 7. - P. 308-313. DOI: $10.1093 /$ comjnl/7.4.308

23. IGBT Modules. Infineon. URL: https://www.infineon.com/cms/en/ product/power/igbt/igbt-modules (дата обращения 08.09.2021).

Поступила 09.09.2021 2.

\section{Информация об авторах}

Прахт В.A., кандидат технических наук, доцент кафедры электротехники и электротехнологических систем Уральского Энергетического Института Уральского Федерального Университета.

Дмитриевский В.А., кандидат технических наук, доцент кафедры электротехники и электротехнологических систем Уральского Энергетического Института Уральского Федерального Университета.

Анучин A.C., доктор технических наук, профессор кафедры автоматизированного электропривода Национального исследовательского университета «МЭИ».

Казакбаев В.M., кандидат технических наук, доцент кафедры электротехники и электротехнологических систем Уральского Энергетического Института Уральского Федерального Университета. 
UDC 621.313 .323

\title{
APPLICATION OF THE NELDER-MEAD METHOD FOR OPTIMIZING A SYNCHRONOUS HOMOPOLAR MOTOR FOR A MINING DUMP TRUCK
}

\author{
Vladimir A. Prakht ${ }^{1}$, \\ va.prakht@urfu.ru
}

Vladimir A. Dmitrievskii', vladimir.dmitrievsky@urfu.ru

\author{
Alecksey S. Anuchin², \\ anuchinas@mpei.ru \\ Vadim M. Kazakbaev 1 , \\ adim.kazakbaev@urfu.ru \\ 1 Ural Federal University, \\ 19, Mira street, Yekaterinburg, 620002, Russia. \\ 2 Moscow Power Engineering Institute, \\ 14, Krasnokazarmennaya street, Moscow, 111250, Russia.
}

The relevance of the study is in the increasing need for the use of mining dump trucks with a diesel-electric (hybrid) drive for the development of minerals. Improving the operational and cost characteristics of the electric drive of mining dump trucks helps to reduce costs in the development of minerals.

The main aim of the study is to find an effective approach to optimizing a synchronous homopolar motor for driving the rear whee/s of a mining dump truck, which makes it possible to solve the problem of the high demand for computing resources when simulating a threedimensional magnetic field of the motor; develop the recommendations for the design of a synchronous homopolar motor with an excitation winding on the stator; apply the optimization to reduce power losses and maximum motor current for a given traction characteristic of the drive, and to reduce the current rating and cost of the semiconductor inverter module of the electric drive of a mining dump truck with the type of motor under consideration.

Object of the research is a design of a six-pole nine-phase synchronous homopolar motor with an excitation winding on the stator with a power rating of $370 \mathrm{~kW}$.

Methods: derivative-free optimization method; equivalent circuit method; mathematical modeling; two-dimensional finite element method. Results. A novel approach to optimization of a synchronous homopolar motor is proposed. This approach is effective from the point of view of the accuracy of calculating the characteristics and computational costs. As a result of optimization, the motor losses and the maximum current required by the motor from the inverter have been significantly reduced. The achieved reduction of the motor current allows reducing the cost of the semiconductor modules of the inverter by 1,4 times (by 2295 United States dollars), and also allows reducing the alternating component in the current of the direct current link of the inverter by the same amount.

\section{Key words:}

Mining dump truck, Nelder-Mead method, synchronous homopolar motor, optimal design, traction motor, traction drives.

The research was performed with the support of the Russian Science Foundation grant (Project No. 21-19-00696).

\section{REFERENCES}

1. Trukhnov L.I. New and promising developments of «BelAZ» - the managing company of the holding «BelAZ-holding» - for miners. Mining information and analytical bulletin (scientific and technical journal), 2015, no. S1-2, pp. 34-41. In Rus.

2. Koellner W., Brown G., Rodriguez J., Pontt J., Cortes P., Miranda H. Recent advances in mining haul trucks. IEEE Transactions on Industrial Electronics, 2004, vol. 51, pp. 321-329. DOI: 10.1109/TIE.2004.825263

3. Kalsi S., Hamilton K., Buckley R.G., Badcock R.A. Superconducting AC Homopolar machines for high-speed applications. Energies, 2019, vol. 12, pp. 1-14. DOI: 10.3390/en12010086

4. Bianchini C., Immovilli F., Bellini A., Lorenzani E., Concari C., Scolari M. Homopolar generators: an overview. Proc. of the 2011 IEEE Energy Conversion Congress and Exposition. Phoenix, USA, 17-22 September 2011. pp. 1523-1527, DOI: $10.1109 /$ /ECE.2011.6063962

5. Dmitrievskii V., Prakht V., Anuchin A., Kazakbaev V. Design optimization of a traction synchronous homopolar motor Mathematics, 2021, vol. 9, pp. 1-14. DOI: 10.3390/math9121352

6. Yu K., Yao J., Xie X., Tang P. A novel critical analysis method of homopolar inductor alternator for preliminary design in capacitor charge power supply. IEEE Transactions on Plasma Science, 2019, vol. 47, no. 5, pp. 2354-2361. DOI: 10.1109/TPS.2019.2892610.

7. Severson E., Nilssen R., Undeland T., Mohan N. Dual-purpose novoltage winding design for the bearingless $\mathrm{AC}$ homopolar and consequent pole motors. IEEE Transactions on Industry Applications, 2015, vol. 51, no. 4, pp. 2884-2895. DOI: 10.1109/TIA.2015.2388852

8. Jeong J.-S., An D.-K., Hong J.-P., Kim H.-J., Jo Y.-S. Design of a 10-MW-Class HTS homopolar generator for wind turbines. IEEE Transactions on Applied Superconductivity, 2017, vol. 27, no. 4, pp. 1-4. DOI: 10.1109/TASC.2017.2669140

9. Bernatt J., Gawron S., Glinka T., Polak A. Traction induction motor. Proc. of the $13^{\text {th }}$ International Conference Modern Electrified Transport (MET). Warsaw, Poland, 5-7 October 2017. pp. 1-5. DOI: $10.1051 /$ matecconf/201818004005

10. Anuchin A.S., Kotelnikov M.K., Ostrirov V.N., Lashkevich M.M., Podlesny M.V., Milsky K.V., Tarasov A.S. Komplekt tyagovogo elektrooborudovaniya dlya BelAZ-90 na baze ventilnoinduktornogo elektrodvigatelya s nezavisimym vozbuzhdeniem [A set of traction electrical equipment for BelAZ-90 based on a synchronous homopolar electric motor with independent excitation]. Trudy X Mezhdunarodnoy konferentsii po 
avtomatizirovannomu elektroprivodu AEP [Proc. of the $\mathrm{X}$ international conference on the automated electric drive AEP 2018]. Novocherkassk, LIK Publ., 2018. pp. 218-224.

11. Cheshmehbeigi H., Afjei E. Design optimization of a homopolar salient-pole brushless DC machine: Analysis, simulation, and experimental tests. IEEE Transactions on Energy Conversion, 2013, vol. 28, no. 2, pp. 289-297. DOI: 10.1109/TEC.2013.2249584.

12. Severson E., Mohan N., Nilssen R., Undeland T. Outer-rotor AC homopolar motors for flywheel energy storage. Proc. of $7^{\text {th }}$ IET Int. Conf. Power Electron., Mach. Drives (PEMD). Manchester, U.K., 2014. pp. 1-6. DOI: $10.1049 /$ cp.2014.0345

13. Dmitrievskii V., Prakht V., Anuchin A., Kazakbaev V. Traction synchronous homopolar motor: simplified computation technique and experimental validation. IEEE Access, 2020, vol. 8, pp. 185112-185120. doi.org/10.1109/ACCESS.2020.3029740

14. Yang J., Ye C., Liang X., Xu W., Xiong F., Xiang Y., Li W. Investigation of a two-dimensional analytical model of the homopolar inductor alternator. IEEE Transactions on Applied Superconductivity, 2018, vol. 28, pp. 1-5. DOI: 10.1109/TASC.2018.2802480

15. Ye C., Yang J., Xiong F., Zhu Z.Q. Relationship between homopolar inductor machine and wound-field synchronous machine. IEEE Transactions on Industrial Electronics, 2020, vol. 67, pp. 919-930. DOI: 10.1109/TIE.2019.2898577

16. Belalahy C., Rasoanarivo I., Sargos F. Using 3D reluctance network for design a three phase synchronous homopolar machine. Proc. of the $200834^{\text {th }}$ Annual Conference of IEEE Industrial Electronics. Orlando, USA, 10-13 November 2008. pp. 20672072. DOI: $10.1109 /$ IECON.2008.4758275

Information about the authors

Vladimir A. Prakht, Cand. Sc., associate professor, Ural Federal University.

Vladimir A. Dmitrievskii, Cand Sc., associate professor, Ural Federal University.

Alecksey S. Anuchin, Dr. Sc., full professor, Moscow Power Engineering Institute.

Vadim M. Kazakbaev, Cand Sc., associate professor, Ural Federal University.
17. FF1000R17IE4, IGBT-Modules, Technical Information, Revision 3.2, Infineon. November 2013. Available at: https://www.infineon.com/dgdl/Infineon-FF1000R17IE4-DSv03_02-EN.pdf?fileId=db3a30431ff9881501201dc994a34980 (accessed 8 September 2021).

18. Prakht V., Dmitrievskii V., Kazakbaev V. Optimal design of gearless flux-switching generator with ferrite permanent magnets. Mathematics, 2020, vol. 8, pp. 1-14. DOI: 10.3390/math8020206

19. FF650R17IE4, IGBT-Modules, Technical Information, Revision 3.3, Infineon. November 2013. Available at: https://www.infineon.com/dgdl/Infineon-FF650R17IE4-DSv03_03-EN.pdf?fileId=db3a30431ff9881501201dcfe2a54986 (accessed 8 September 2021).

20. Cupertino F., Pellegrino G., Gerada C. Design of synchronous reluctance machines with multiobjective optimization algorithms. IEEE Transactions on Industry Applications, 2014, vol. 50, pp. 3617-3627. DOI: 10.1109/TIA.2014.2312540

21. Krasopoulos C.T., Beniakar M.E., Kladas A.G. Robust optimization of high-speed PM motor design. IEEE Transactions on Magnetics, 2017, vol. 53, pp. 1-4. DOI: 10.1109/TMAG.2017.2660238

22. Nelder J.A., Mead R.A. Simplex method for function minimization. The Computer Journal, 1965, vol. 7, pp. 308-313. DOI: $10.1093 /$ comjnl/7.4.308

23. IGBT Modules. Infineon. Available at: https://www.infineon.com/ cms/en/product/power/igbt/igbt-modules (accessed 8 September 2021)

Received: 09 September 2021. 